\title{
Compressible 1-D Euler Equations with Large Data: A Case Study
}

\author{
Erik Endres* $\quad$ Helge Kristian Jenssen ${ }^{\dagger}$
}

April 23, 2008

\begin{abstract}
Consider one-dimensional flow of a compressible, ideal, and polytropic gas on a bounded interval in Lagrangian variables. We study the Cauchy problem when the initial data consist of four constant states that yield two contact waves bounding an interval of lower density, together with an admissible shock between them. To render the solution tractable for direct calculations we also impose absorbing boundary conditions, at fixed locations (in Lagrangian coordinates) and depending on the data, to the left and to the right of the two contacts. By estimating the wave strengths in shock-contact interactions we show that the resulting flow is defined for all times. In particular, the pressure, density, particle velocities and shock speeds are all uniformly bounded in time. We also record a scaling invariance of the system and comment on its relevance to large data solutions of the Euler system.
\end{abstract}

Keywords: Initial-boundary value problem for gas dynamics, global in time weak solutions. 2000 Mathematics Subject Classification: 35L65, 35L67, 35L45, $76 N 10$.

\section{Contents}

1 Introduction $\quad 2$

2 Shock-contact interactions $\quad 4$

3 Properties of the interaction pattern $\quad 5$

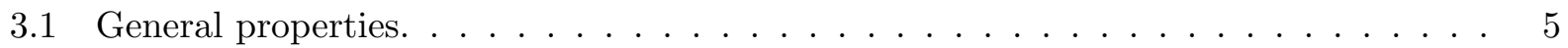

3.2 Properties assuming pressure is bounded. . . . . . . . . . . . . 7

4 Boundedness of pressure

4.1 Parametrization of shock curves. . . . . . . . . . . . . . . . 8

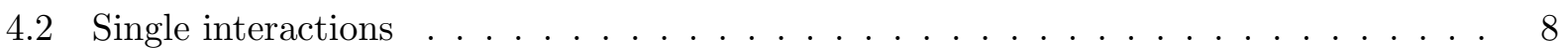

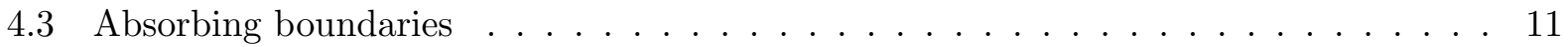

4.4 Interaction pattern $\ldots \ldots \ldots \ldots \ldots \ldots \ldots \ldots$

5 Scaling invariance of the 1-D Euler system 14

\footnotetext{
*Department of Mathematics, Penn State University, University Park, State College, PA 16802, USA (endres@math.psu.edu)

${ }^{\dagger}$ Department of Mathematics, Penn State University, University Park, State College, PA 16802, USA (jenssen@math.psu.edu). Partially supported by NSF grant DMS-0539549.
} 


\section{Introduction}

We consider the 1-D Euler system describing conservation of mass, momentum, and energy in compressible, inviscid, and adiabatic gas flow:

$$
\begin{aligned}
\tau_{t}-u_{x} & =0 \\
u_{t}+p_{x} & =0 \\
E_{t}+(p u)_{x} & =0 .
\end{aligned}
$$

Here $x$ is a Lagrangian space variable, $u$ is velocity, $p$ is pressure, $\tau$ is specific volume, $E=e+u^{2} / 2$ is specific total energy, and $e$ is internal energy. The density $\rho$ is given by $\rho=1 / \tau$.

For initial data with sufficiently small total variation Glimm's theorem [11] applies and guarantees existence of a global-in-time weak, entropy admissible solution. The solution can be constructed by various methods: Glimm's original random choice method [11], Liu's deterministic version [14], the wave-front tracking schemes of DiPerna [9], Bressan [5], and Risebro [21], or the more recent wave tracing methods of Bianchini and Bressan [4], [2], [3].

The Euler system plays a distinguished role among hyperbolic systems and much effort has been invested in extending Glimm's existence result to larger classes of data. For "generic" data the solutions exhibit complicated wave patterns a myriad of interactions.

For the $2 \times 2$-system of isothermal gas dynamics, i.e. (1) and (2) with $p=p(\tau)=a^{2} / \tau$ (disregarding (3)), Nishida [17] established global existence for any bounded $\mathrm{BV}_{l o c}$ data. The key property in this particular case is the translation invariance of wave curves. See [24] for a generalization to $2 \times 2$-systems with this property. Nishida's result initiated a series of related results for more general $2 \times 2$-systems, [1], [8], [18]. For example, for isentropic gas dynamics $((1)$ and (2) with $p=p(\tau)=a^{2} / \tau^{\gamma}, \gamma>1$, and disregarding (3)), Nishida and Smoller [18] established existence for possibly large data that satisfy

$$
(\gamma-1) \cdot(\text { Total variation of data }) \quad \text { is sufficiently small. }
$$

The same authors extended this type of results to the mixed initial-boundary value problem for isentropic gas dynamics [19]. In particular they treated the case of a "double-piston" and provided conditions on the data ((4) together with strict separation of the pistons) that guarantee existence of global weak solutions.

Liu [15], [16] provided a significant extension of these results to the full Euler system (1)-(3). For the Cauchy problem for an ideal polytropic gas with equation of state

$$
p=p(\tau, S)=a^{2} \tau^{-\gamma} \exp [(\gamma-1) S / R],
$$

where $S$ denotes entropy and $\gamma \in(1,5 / 3$ ], existence of a global solution is shown in [15] under the condition that

$$
(\gamma-1) \cdot[\text { T.V.u }(x, 0)+\text { T.V. } \tau(x, 0)+\text { T.V.S }(x, 0)] \quad \text { is sufficiently small. }
$$

Here T.V. denotes total variation. In [16] Liu extended this result to the initial-boundary problem and he also showed that for the initial value problem, (5) may be replaced by the condition

$$
(\gamma-1) \cdot[\text { T.V.u } u(x, 0)+\text { T.V.p }(x, 0)] \quad \text { is sufficiently small. }
$$


This last result was extended to any $\gamma>1$ by Peng [20]. Temple [26] considered more general 1-parameter families of gas dynamical equations of state $e_{\varepsilon}(\tau, S)$ with the property that

$$
e_{0}(\tau, S)=-\ln \tau+S / R
$$

A generalization of Liu's result for the Cauchy problem was established through a careful analysis where also the role of $\varepsilon$ is recorded.

Temple and Young [27] have established existence for the full Euler system (and more generally for $3 \times 3$-systems with a Riemann invariant) up to an arbitrary time for data with large total variation and sufficiently small sup-norm. In an interesting recent work Edens [10] has considered linearly degenerate models for gas dynamics (introduced earlier by Temple and Young) where the interaction coefficients have the same signs as for compressible Euler. Special solutions are constructed where waves interact at regularly spaced grid points. An analysis of the spectral properties of the map which updates the strengths of 1 and 3 waves at interactions demonstrates decay of total variation. We mention finally a result of Rykov [23] who considered general BV data. Provided one has an a priori bound on the $L^{\infty}$ norm of approximate solutions (generated by Glimm's scheme, say), and provided the approximations stay bounded away from vacuum, then the total variation of the approximations remains uniformly bounded.

Given the general form of Glimm's theorem it is natural to consider other systems of conservation laws and to investigate various large data regimes for these. However, recent examples show that no result similar to Glimm's theorem can hold at this level of generality. In particular [12], [13], [28] give examples of special (unphysical) systems of conservation laws whose solutions may suffer blowup of amplitude and/or total variation. It is not known if similar pathological behavior can occur for physical systems. Such systems are typically equipped with a convex entropy and one could hope that this additional structure would prevent this type of breakdown. The most interesting system in this respect is the Euler system.

The blowup examples in [12], [28] were based on particular interaction patterns where repeated reflections create infinitely many waves in finite time. By judiciously choosing the initial data one can generate solutions where wave strengths are amplified at each interaction, leading to finite time blowup.

In this paper we construct special solutions of the full Euler system with a particular interaction pattern of the same type as in [12], [28]. We then investigate if such solutions can produce growth, or even finite time blowup, of amplitudes or variation. We consider the case of an ideal and polytropic gas where shocks undergo repeated reflections as they interact with two approaching contact waves. Our analysis shows that no blowup occurs for solutions of this type, at least when the transmitted shock waves are absorbed and thus prevented from interfering with the basic pattern of shocks and contacts, see Figure 1. Imposing absorbing boundary conditions outside the central region amounts to disregarding interactions of shocks in the same family and renders the analysis tractable.

We begin by setting up the specific interaction pattern and deriving some of its properties in sections 2 and 3. Assuming an upper bound on the pressure we show that the solution is globally defined and remains uniformly bounded. The main part of the analysis concerns the uniform bound on the pressure (section 4). We also describe briefly how to prescribe absorbing boundary conditions. The conclusion of our analysis is recorded in Proposition 4.3. Finally, in section 5 we comment briefly on a particular scaling of the dependent variables in the Euler system which provides a (weak) large data result for certain types of "scaled up" data. 


\section{Shock-contact interactions}

For the background material in this section we refer to [7], [6], [22], [25]. For an ideal polytropic gas the equation of state is

$$
e=\frac{\tau p}{\gamma-1}, \quad \gamma>1
$$

and the pressure is given by

$$
p=\frac{K \theta}{\tau},
$$

where $\theta$ denotes temperature and $K /(\gamma-1)$ is the specific heat of the gas at constant volume. For a given left state $\bar{U}=\left(\tau_{0}, \bar{u}, \bar{p}\right)$, the set of states that can be connected to $\bar{U}$ on the right through an entropy admissible backward or forward shock are given by

$$
\begin{aligned}
\tau & =\frac{\bar{\tau}\left(\bar{p}+\mu^{2} p\right)}{p+\mu^{2} \bar{p}} \\
u & =\bar{u} \pm(p-\bar{p}) \sqrt{\frac{\left(1-\mu^{2}\right) \bar{\tau}}{p+\mu^{2} \bar{p}}} \quad \begin{cases}+ \text { : forward shock } \vec{S} & \bar{p}>p, \\
- \text { : backward shock } \overleftarrow{S} & \bar{p}<p .\end{cases}
\end{aligned}
$$

with corresponding speeds given by

$$
\sigma_{ \pm}= \pm \sqrt{\frac{p-\bar{p}}{\bar{\tau}-\tau}}
$$

Here

$$
\mu^{2}=\frac{\gamma-1}{\gamma+1}
$$

The Euler system also allows contact discontinuities across which the pressure and velocity are continuous, while the density jumps:

$$
u=\bar{u}, \quad p=\bar{p}, \quad \bar{\tau} \gtrless \tau \quad\left\{\begin{array}{l}
>\text { : down-contact } \vec{J}, \\
<: \text { up-contact } \hat{J} .
\end{array}\right.
$$

A calculation shows (see [6] pp. 110-114) that the interaction of a backward shock with an upcontact results in a transmitted backward shock, an up-contact, and a reflected forward shock:

$$
\overleftarrow{J S} \mapsto \overleftarrow{S}\langle\vec{S}
$$

Similarly,

$$
\vec{S} \vec{J} \mapsto \overleftrightarrow{S} \vec{J} \vec{S}
$$

These interactions will be used to set up an interaction pattern where reflected shocks traverse an inner region between two contacts where the density is lower than in the outside regions; see Figure 1. As a consequence of Lax' entropy inequalities the transmitted shocks in the outer regions will approach each other. In an interaction between two shocks of the same family the type (shock/rarefaction) of the reflected wave depends on the value of $\gamma$, and may also depend on their relative strengths. In either case the wave reflected from these same-family interactions would 


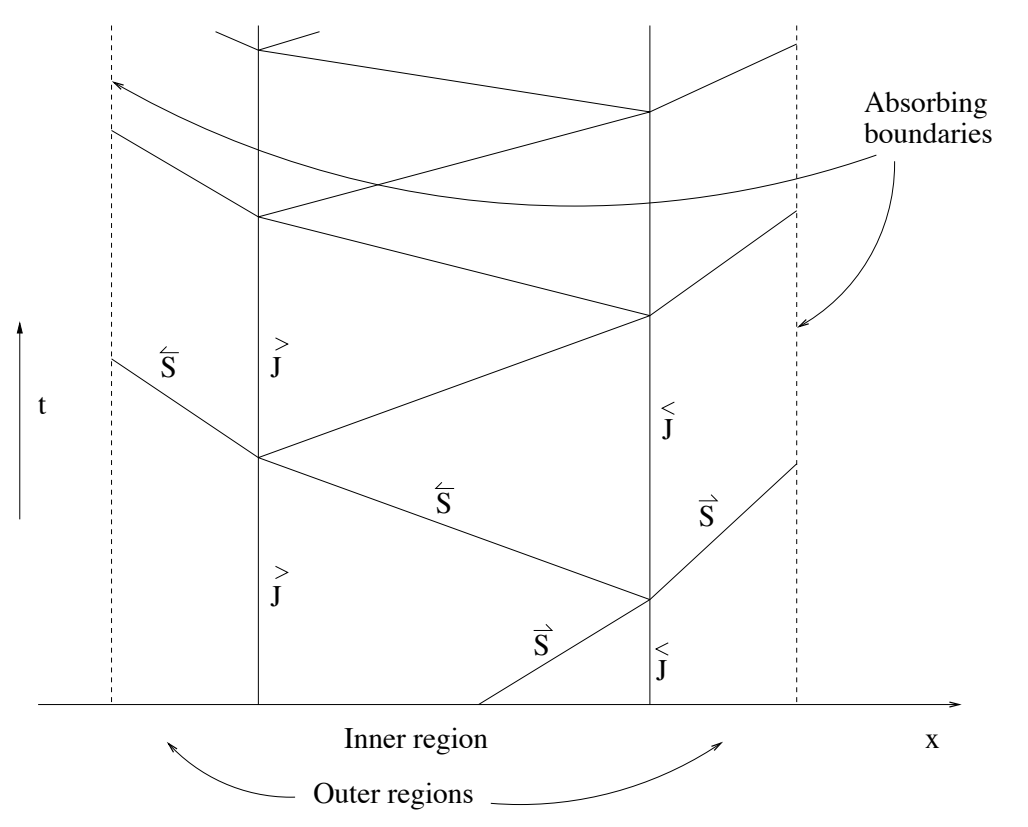

Figure 1: Interaction pattern with repeated reflections.

interact either with the "next" transmitted shock and/or with a contact. The resulting pattern quickly becomes complicated and does not seem amenable to a direct analysis. In order to render the time evolution computationally tractable we avoid this issue altogether by inserting absorbing boundaries in the outer regions (along the dotted lines in Figure 1). While it is clear from (12) and (13) that the resulting pattern (now with boundary conditions imposed) will only involve forward and backward shocks together with the two contacts, it is not immediately clear that it can be continued indefinitely in time. Specifically we need to show that there are only a finite number of interactions in finite time. To this end we show that the speeds of all reflected shocks in the solution are uniformly bounded in time. A similar bound on the speeds of the transmitted shocks shows that we can impose the boundary conditions at fixed locations (in Lagrangian coordinates), as indicated in Figure 1.

In the next section derive some general properties on the densities, pressures and velocities in the outer and inner regions. We then show that, provided the pressures in the three regions remain uniformly bounded, then shocks speeds, particle velocities, densities, as well as specific volumes, all remain uniformly bounded. In the final step we show that pressure is indeed uniformly bounded.

\section{Properties of the interaction pattern}

\subsection{General properties.}

In what follows we use superscripts $L$ and $R$ to denote states in the outer left and right regions, respectively. For the inner region we use superscripts $M$ and $N$; the various constant states resulting from interactions are indexed as in Figure 2.

Since shocks are compressive (i.e. a particle experiences an increase in pressure and density as 


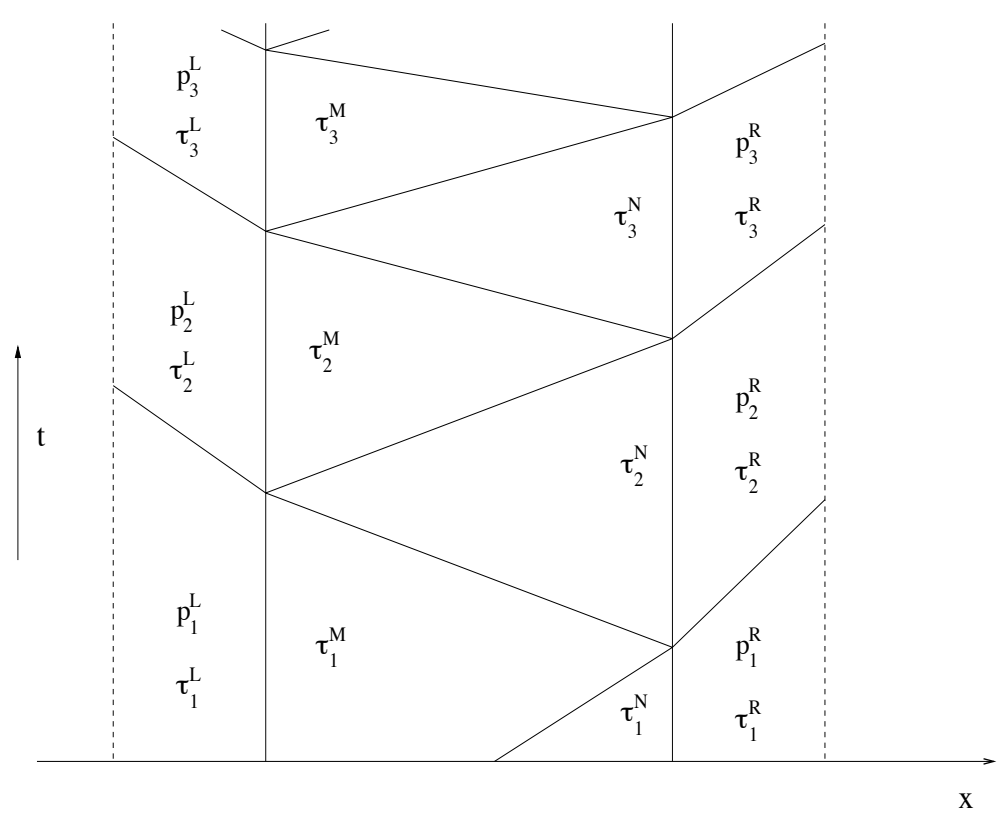

Figure 2: Interaction pattern with states resulting from interactions.

it crosses a shock, [7]) we obtain the following series of inequalities:

$$
\begin{aligned}
& \tau_{1}^{L}>\tau_{2}^{L}>\cdots, \\
& \tau_{1}^{R}>\tau_{2}^{R}>\cdots \\
& \tau_{1}^{N}>\tau_{1}^{M}>\tau_{2}^{N}>\tau_{2}^{M}>\cdots .
\end{aligned}
$$

Each sequence is decreasing and bounded below, and thus convergent. Furthermore, the density in each region is bounded away from vacuum. Compressibility also gives that

$$
p_{1}^{R}<p_{1}^{L}<p_{2}^{R}<p_{2}^{L}<\cdots .
$$

As velocity and pressure do not change across contacts we have

$$
u_{i}^{L}=u_{i}^{M}, \quad p_{i}^{L}=p_{i}^{M}, \quad u_{i}^{R}=u_{i}^{N}, \quad p_{i}^{R}=p_{i}^{N} .
$$

By (17) and (9) we have

$$
u_{i+1}^{L}-u_{i}^{L}=-\left(p_{i+1}^{L}-p_{i}^{L}\right) \sqrt{\frac{\left(1-\mu^{2}\right) \tau_{i}^{L}}{p_{i+1}^{L}+\mu^{2} p_{i}^{L}}}<0,
$$

such that

$$
u_{1}^{L}>u_{2}^{L}>\cdots .
$$

Similarly one finds for the outer right region and for the inner region that

$$
u_{1}^{R}<u_{2}^{R}<\cdots, \quad \text { and } u_{i}^{R}<u_{i}^{L} \quad \forall i, \quad \text { and in fact } u_{i}^{R}<u_{j}^{L} \quad \forall i, j .
$$

It follows that $\left\{u_{i}^{L}\right\}$ is a decreasing and bounded sequence, while $\left\{u_{i}^{R}\right\}$ is an increasing and bounded sequence. Thus they both converge. 
Next we show that the temperature, $\theta=K^{-1} p \tau$, increases in each region. Consider a shock in any of the three regions and let $p_{i}, \tau_{i}, \theta_{i}$ and $p_{i+1}, \tau_{i+1}, \theta_{i+1}$ be the pressure, specific volume, and temperature of a fluid particle before and after passing through the shock, respectively. The relation between pressures and specific volumes is given by (8). The relationship is symmetric in $p$ and $\bar{p}$ and thus takes the same form for forward and for backward shocks. Since $p_{i+1}>p_{i}$, equation (8) yields

$$
\begin{aligned}
\theta_{i+1}=K^{-1} p_{i+1} \tau_{i+1} & =K^{-1} p_{i+1} \frac{\tau_{i}\left(p_{i}+\mu^{2} p_{i+1}\right)}{\left(p_{i+1}+\mu^{2} p_{i}\right)} \\
& >K^{-1} p_{i+1} \frac{\tau_{i}\left(p_{i}+\mu^{2} p_{i}\right)}{\left(p_{i+1}+\mu^{2} p_{i+1}\right)}=K^{-1} p_{i} \tau_{i}=\theta_{i}
\end{aligned}
$$

\subsection{Properties assuming pressure is bounded.}

For the following calculations we assume that pressure is uniformly bounded above. This is then established in section 4 , independently of the results in this subsection.

If the pressure is bounded above then (17) shows that the pressure in each region converges to a common value $\bar{p}$ :

$$
\bar{p}:=\lim _{i \rightarrow \infty} p_{i}^{L}=\lim _{i \rightarrow \infty} p_{i}^{R} .
$$

We proceed to use this to bound the other flow variables in the three regions. For concreteness consider the outer left region. From (19), and using that $\tau_{i}^{L}$ is a convergent sequence by (14), we find that

$$
0<p_{1}^{L} \tau_{1}^{L}<\lim _{i \rightarrow \infty}\left(p_{i}^{L} \tau_{i}^{L}\right)=\bar{p} \cdot\left(\lim _{i \rightarrow \infty} \tau_{i}^{L}\right),
$$

which shows that $\tau_{i}^{L}$ converges to a finite positive value. A similar analysis applies in the other regions. Therefore there is a $\bar{\tau}>0$ such that

$$
\tau_{i}^{L}, \tau_{i}^{R}, \tau_{i}^{N}, \tau_{i}^{M}>\bar{\tau} \quad \forall i
$$

Next consider the speed of shock waves in any of the three regions. Let $p_{i}, \tau_{i}$ and $p_{i+1}, \tau_{i+1}$ be the pressure and specific volume of a fluid particle before and after passing through a shock, respectively. By (8) and (10) the shock speed $\sigma_{i}$ is given by

$$
\left|\sigma_{i}\right|=\sqrt{\frac{p_{i+1}-p_{i}}{\tau_{i}-\tau_{i+1}}}=\sqrt{\frac{p_{i+1}+\mu^{2} p_{i}}{\tau_{i}\left(1-\mu^{2}\right)}} .
$$

Since $p_{i}, p_{i+1}<\bar{p}$ and $\tau_{i}>\bar{\tau}$ we have

$$
\left|\sigma_{i}\right|<\sqrt{\frac{\bar{p}+\mu^{2} \bar{p}}{\bar{\tau}\left(1-\mu^{2}\right)}}=\sqrt{\frac{\bar{p} \gamma}{\bar{\tau}}} \quad \forall i .
$$

This shows that all shock speeds are uniformly bounded. Therefore, on any finite time interval there is only a finite number of shock-contact interactions. It follows that the interaction pattern exists for all times, and that $p, \tau$ and $u$ converge to finite values in all regions. Notice that the specific volume is uniformly bounded away from 0 as well as away from $+\infty$ (no vacuum occurs). As shock speeds are bounded it also follows that the absorbing boundaries in the outer regions may be prescribed at fixed locations (in Lagrangian coordinates). 


\section{Boundedness of pressure}

\subsection{Parametrization of shock curves.}

To investigate how the pressure increases in the solution we parametrize shocks by pressure ratios $p_{+} / p_{-}$, where $p_{+}, p_{-}$denote pressure on the right and on the left, respectively. Similarly, contacts are parametrized by the ratio of specific volumes. For a forward shock we denote the pressure ratio by $f$, and for a backward shock we denote the pressure ratio by $b$. Since fuid particles experience an increase in pressure when crossing a shock we have $b>1$ and $f<1$. For contacts we denote the specific volume ratio by $c$. This provides the following parametrization of shock-curves and contact-curves in $(\tau, u, p)$-space centered at $(\bar{\tau}, \bar{u}, \bar{p})$ (cf. [25], [6]):

$$
\begin{aligned}
& \overleftarrow{S}(b ; \bar{\tau}, \bar{u}, \bar{p})=\left(\begin{array}{c}
\phi(b) \bar{\tau} \\
\bar{u}-\psi(b) \sqrt{\bar{\tau} \bar{p}} \\
b \bar{p}
\end{array}\right), \quad b>1, \\
& \underset{J}{\lessgtr}(c ; \bar{\tau}, \bar{u}, \bar{p})=\left(\begin{array}{c}
c \bar{\tau} \\
\bar{u} \\
\bar{p}
\end{array}\right), \quad 1 \lessgtr c, \\
& \vec{S}(f ; \bar{\tau}, \bar{u}, \bar{p})=\left(\begin{array}{c}
\phi(f) \bar{\tau} \\
\bar{u}+\psi(f) \sqrt{\bar{\tau} \bar{p}} \\
f \bar{p}
\end{array}\right), \quad f<1,
\end{aligned}
$$

where, for $\alpha \geq 0$,

$$
\phi(\alpha)=\frac{1+\mu^{2} \alpha}{\alpha+\mu^{2}}, \quad \psi(\alpha)=\frac{\kappa(\alpha-1)}{\sqrt{\alpha+\mu^{2}}}, \quad \kappa=\sqrt{1-\mu^{2}} .
$$

We note that the function $\psi(\alpha)$ is smooth, strictly increasing, concave down (see Figure 3), and satisfies the relation

$$
\sqrt{\alpha \phi(\alpha)} \psi\left(\frac{1}{\alpha}\right)=-\psi(\alpha)
$$

\subsection{Single interactions}

Consider the Riemann problem that occurs when a forward shock interacts with a down-contact. Let the outgoing forward and backward shocks have pressure ratios $F$ and $B$, respectively, and let $C$ denote the ratio of specific volumes across the outgoing contact. See Figure 4. From (12) and (13) we thus have $F<1, B>1$, and $C \gtrless 1$ if and only if $c \gtrless 1$. Traversing the various waves before

and after interaction, and using (24)-(26), yield the following relationships between incoming and outgoing ratios:

$$
\begin{aligned}
c \phi(f) & =C \phi(B) \phi(F) \\
\psi(f) & =\psi(F) \sqrt{C B \phi(B)}-\psi(B) \\
f & =B F .
\end{aligned}
$$

Eliminating $F$ and $C$, and using (28), one obtains the following equation for the pressure ratio across the reflected (backward, outgoing) wave:

$$
\psi(B)+\sqrt{c f \phi(f)} \psi\left(\frac{B}{f}\right)+\psi(f)=0 .
$$




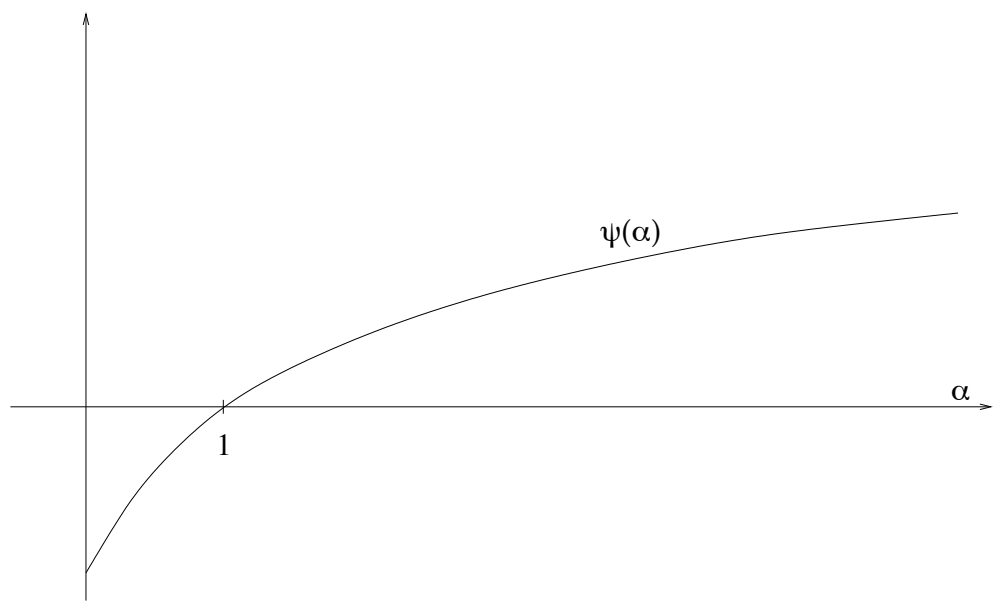

Figure 3: Graph of the function $\psi$ (schematic)

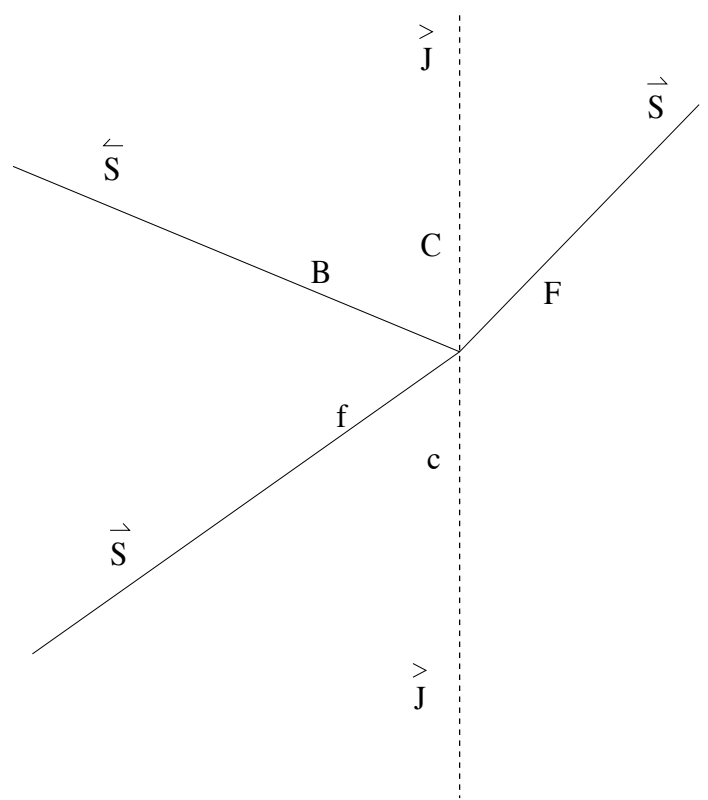

Figure 4: Interaction of a forward shock with a down contact 
Define $\mathcal{F}(\alpha ; f, c)$ to be the expression on the left-hand side of $(32)$ :

$$
\mathcal{F}(\alpha ; f, c):=\psi(\alpha)+\sqrt{c f \phi(f)} \psi\left(\frac{\alpha}{f}\right)+\psi(f) .
$$

Since $\psi$ is an increasing function it follows that $\alpha \mapsto \mathcal{F}(\alpha ; f, c)$ is an increasing function for fixed values of $f$ and $c$. As $F(0 ; f, c)<0$ it follows that (32) has a unique root $B$. That is,

the outgoing pressure ratio $B$ is the unique root of $\quad \alpha \mapsto \mathcal{F}(\alpha ; f, c)$.

(This calculation provides one part of the analysis behind the statements in (12) and (13). See [6] for further details). We proceed to estimate $B$ in terms of the incoming waves. Consider

$$
\mathcal{F}\left(\frac{1}{f} ; f, c\right)=\psi\left(\frac{1}{f}\right)+\sqrt{c f \phi(f)} \psi\left(\frac{1}{f^{2}}\right)+\psi(f) .
$$

Since $f<1$ we have $\psi\left(\frac{1}{f^{2}}\right)>0$, while $(27)$ and (28) shows that

$$
\psi\left(\frac{1}{f}\right)+\psi(f)=\psi\left(\frac{1}{f}\right)[1-\sqrt{f \phi(f)}]>0,
$$

giving

$$
\mathcal{F}\left(\frac{1}{f} ; f, c\right)>0
$$

Since $\mathcal{F}(\cdot ; f, c)$ is an increasing function with a unique zero $B$ we conclude that

$$
B<\frac{1}{f} .
$$

Next consider the ratio

$$
\frac{c}{C}=\frac{\phi(B) \phi(F)}{\phi(f)}
$$

Evaluating the $\phi$ 's and using that $B=\frac{f}{F}$ we find that $c<C$ if and only if

$$
\left(F+\mu^{2} f\right)\left(1+\mu^{2} F\right)\left(f+\mu^{2}\right)<\left(f+\mu^{2} F\right)\left(1+\mu^{2} f\right)\left(F+\mu^{2}\right) .
$$

Expanding the products and using that $F<f<1$ (since $B>1$ ), one finds that the inequality (38) is satisfied, and hence

$$
c<C<1 \text {. }
$$

Next consider a backward shock interacting with an up-contact. This is exactly the mirror image of the previous case. Denote the incoming backward shock strength by $b^{\prime}$, incoming contact by $c^{\prime}$, and the outgoing strengths by $B^{\prime}, C^{\prime}, F^{\prime}$. Then, with $f=1 / b^{\prime}, c=1 / c^{\prime}, B=1 / F^{\prime}, C=1 / C^{\prime}$, and $F=1 / B^{\prime}$, we have the same result as above. This shows that

$$
F^{\prime} \quad \text { is the unique root of } \quad \alpha \mapsto \mathcal{F}\left(\frac{1}{\alpha} ; \frac{1}{b^{\prime}}, \frac{1}{c^{\prime}}\right)
$$

and that

$$
\frac{1}{F^{\prime}}<b^{\prime}, \quad c^{\prime}>C^{\prime}>1 .
$$




\subsection{Absorbing boundaries}

A similar analysis of shocks interacting with contact discontinuities between gases with different adiabatic exponents $\gamma$ shows the following. Consider a forward shock with pressure ratio $f<$ 1 interacting with a contact discontinuity with specific volume ratio $c \neq 1$. Let the adiabatic exponents on the left and right of the contact be $\gamma_{0}, \gamma_{1}$, respectively. By resolving the Riemann problem at the interaction as above one finds that no wave is reflected if and only if

$$
\gamma_{1}=c \gamma_{0}+(c-1) \frac{1-f}{1+f}
$$

We only consider gases whose adiabatic exponents are stricly larger than 1 , and we observe that $\gamma_{1}$ given by (41) satisfies $\gamma_{1}>1$ if and only if

$$
c>\frac{2}{\gamma_{0}(1+f)+(1-f)}=: c^{*},
$$

where $c^{*}<1$. Therefore, for given values of $\gamma_{0}$ and $f$, in order to have no backward outgoing wave from the interaction, we may first choose any ratio $c \in\left(c^{*}, 1\right) \cup(1, \infty)$, and then choose $\gamma_{1}$ according to (41). Having done so, we find that the ratio $C$ of specific volumes across the outgoing contact is given by

$$
C=\frac{c \gamma_{0}(1+f)-(2-c)(1-f)}{\gamma_{0}(1+f)-(1-f)}
$$

Notice, $C \gtrless 1$ if and only if $c \gtrless 1$.

We proceed to construct an absorbing right boundary for our solution. As above we let $\gamma$ denote the adiabatic exponent in the region between the left and right boundaries, and we define the pressure ratios in the outer right region by

$$
F_{i}:=\frac{p_{i}^{R}}{p_{i+1}^{R}} .
$$

The strength of the contacts at the right boundary will be chosen as a common constant $c_{1}>1$. Before the first forward shock interacts with the boundary we choose an adiabatic exponent $\gamma_{1}$ on the right of the boundary to be

$$
\gamma_{1}:=c_{1} \gamma+\left(c_{1}-1\right) \frac{1-F_{1}}{1+F_{1}}
$$

The specific volume to the right of the boundary is given by

$$
\tau_{1}=c_{1} \tau_{1}^{R} \text {. }
$$

By construction this will absorb the incoming forward shock. After the interaction we change the adiabatic exponent on the right of the boundary to the new value

$$
\gamma_{2}:=c_{1} \gamma_{0}+\left(c_{-} 1\right) \frac{1-F_{2}}{1+F_{2}} .
$$

The specific volume to the right of the boundary is now given by

$$
\tau_{2}=c_{1} \tau_{2}^{R} \text {. }
$$

This ensures that the next incoming shock is absorbed without reflection, and we may continue the process. Notice that all $\gamma_{i}$ are bounded between $\gamma$ and $c_{1}(\gamma+1)$. Similarly, by (15), (22) and the fixed choice of $c_{1}$, the specific volumes to the right of the boundary remain uniformly bounded above, as well as away from zero. A similar analysis holds for the left boundary. 


\subsection{Interaction pattern}

We proceed to use the estimates derived above to bound the pressure that results from multiple reflections in the inner region. We define the pressure ratios for the inner region and specific volume ratios across contacts by

$$
f_{k}:=\frac{p_{k}^{R}}{p_{k}^{L}}, \quad b_{k}:=\frac{p_{k+1}^{R}}{p_{k}^{L}}, \quad c_{k}^{L}:=\frac{\tau_{k}^{M}}{\tau_{k}^{L}}, \quad c_{k}^{R}:=\frac{\tau_{k}^{R}}{\tau_{k}^{N}},
$$

see Figure 2. Assume that we have resolved the $k-1$ first interactions on the right as well as the $k-1$ interactions on the left, thus knowing the ratios $f_{k}, c_{k}^{R}$, and $c_{k}^{L}$. As detailed above, resolving the next two interactions then gives:

- first, the ratios $b_{k}$ and $c_{k+1}^{R}$, from the $k$ th interaction on the right,

- and then the ratios $f_{k+1}$ and $c_{k+1}^{L}$, from the $k$ th interaction on the left,

where we recall that

$$
b_{k} \quad \text { is the unique root of } \quad \alpha \mapsto \mathcal{F}\left(\alpha ; f_{k}, c_{k}^{R}\right) \text {, }
$$

and that

$$
f_{k+1} \quad \text { is the unique root of } \quad \alpha \mapsto \mathcal{F}\left(\frac{1}{\alpha} ; \frac{1}{f_{k}}, \frac{1}{c_{k}^{L}}\right) .
$$

From the estimates in (37) and (40) we get monotone sequences

$$
\begin{aligned}
& \frac{1}{f_{1}}>b_{1}>\frac{1}{f_{2}}>b_{2} \cdots>1, \\
& c_{1}^{R}<c_{2}^{R}<\cdots<1, \\
& c_{1}^{L}>c_{2}^{L}>\cdots>1 .
\end{aligned}
$$

The key estimate we use to bound the pressure is contained in the following lemma.

Lemma 4.1. Given the ratio $c_{1}^{R}$ of specific volumes across the initial contact on the right. Then there exists $\xi=\xi\left(c_{1}^{R}\right)$ with $0<\xi<1$ and such that

$$
b_{k}<(1-\xi)+\frac{\xi}{f_{k}} \quad \text { for all } k \geq 1 .
$$

Proof. Since $\alpha \mapsto \mathcal{F}(\alpha ; f, c)$ is increasing for fixed $f$ and $c$, and by (42), we have that (47) holds if and only if

$$
0<\mathcal{F}\left((1-\xi)+\frac{\xi}{f_{k}} ; f_{k}, c_{k}^{R}\right) .
$$

By concavity of $\alpha \mapsto \mathcal{F}(\alpha ; f, c)$, and assuming that $\xi \in(0,1)$, (48) follows provided

$$
0<(1-\xi) \mathcal{F}\left(1 ; f_{k}, c_{k}^{R}\right)+\xi \mathcal{F}\left(\frac{1}{f_{k}} ; f_{k}, c_{k}^{R}\right)
$$

Next, using that $c_{k}^{R}>c_{1}^{R}$ and that both $z \mapsto \mathcal{F}\left(1 ; f_{k}, z\right)$ and $z \mapsto \mathcal{F}\left(\frac{1}{f_{k}} ; f_{k}, z\right)$ are increasing (since $f_{k}<1$, see $\left.(33)\right)$, we have that (49) holds provided

$$
0<(1-\xi) \mathcal{F}(1 ; f, c)+\xi \mathcal{F}\left(\frac{1}{f} ; f, c\right),
$$


where we have set $c=c_{1}^{R}$ and $f=f_{k}$ for convenience. We proceed to show that there exists $\xi=\xi(c) \in(0,1)$ such that $(50)$ holds for all $f<1$. Indeed, using the explicit expressions for the functions $\mathcal{F}$ and $\psi$, together with the relationship (28), we have that (50) holds if and only if

$$
0<(1-\xi)(\sqrt{c}-1)|\psi(f)|+\xi\left\{\psi\left(\frac{1}{f}\right)+\sqrt{c} \frac{\psi\left(1 / f^{2}\right)}{\psi(1 / f)}|\psi(f)|-|\psi(f)|\right\} .
$$

Since $\psi$ is increasing and $f<1,(51)$ holds provided we have

$$
0<(1-\xi)(\sqrt{c}-1)|\psi(f)|+\xi\left\{\psi\left(\frac{1}{f}\right)+(\sqrt{c}-1)|\psi(f)|\right\}
$$

or, equivalently,

$$
\frac{1-\sqrt{c}}{\xi}<\frac{\psi\left(\frac{1}{f}\right)}{|\psi(f)|}=\sqrt{\frac{f+\mu^{2}}{f+f^{2} \mu^{2}}} .
$$

Since $f<1$, (53) holds if $1-\sqrt{c}<\xi$. This shows that $\xi=\xi(c):=1-\sqrt{c} / 2$ satisfies the conclusion of the lemma.

For interactions on the left we have a similar result:

Lemma 4.2. Given the ratio $c_{1}^{L}$ of specific volumes across the initial contact on the left. Then there exists $\xi=\xi\left(c_{1}^{L}\right)$ with $0<\xi<1$ and such that

$$
\frac{1}{f_{k+1}}<(1-\xi)+\xi b_{k} \quad \text { for all } k \geq 1
$$

Proof. As in the proof of the Lemma 4.1 we use (43) to see that (54) is equivalent to having

$$
0<\mathcal{F}\left((1-\xi)+\xi b_{k} ; \frac{1}{b_{k}}, \frac{1}{c_{k}^{L}}\right) .
$$

Setting $f_{k}^{\prime}=\frac{1}{b_{k}}$ and $c_{k}^{R^{\prime}}=\frac{1}{c_{k}^{L}}$ we have that (55) takes exactly the same form as (48), and the same argument as above yields the results.

We proceed to use these lemmas to bound the pressure. Consider the $k$ th interaction on the right where a forward shock with pressure ratio $f_{k}$ meets a down-contact and set

$$
x_{k}:=\frac{1}{f_{k}}-1>0 .
$$

By (44) the $x_{k}$ form a decreasing sequence of positive numbers, and by Lemma 4.1 there exists a fixed $0<\xi^{R}<1$ such that

$$
b_{k}<1+x_{k} \xi^{R}, \quad \text { for all } k \geq 1 .
$$

Next consider the $k$ th interaction on the the left where a backward shock with pressure ratio $b_{k}$ meets an up-contact and set

$$
\hat{x}_{k}:=b_{k}-1>0 .
$$

By (44) the $\hat{x}_{k}$ form a decreasing sequence of positive numbers, and by Lemma 4.2 there exists a fixed $0<\xi^{L}<1$ such that

$$
\frac{1}{f_{k+1}}<1+\hat{x}_{k} \xi^{L}, \quad \text { for all } k \geq 1 \text {. }
$$


Set $\xi:=\max \left(\xi^{R}, \xi^{L}\right)<1$, which according to Lemma 4.1 and Lemma 4.2 only depends on the ratios of specific volumes across the two initial contacts. Using the above definitions and inequalities we thus have:

$$
\begin{aligned}
1+x_{1}= & \frac{1}{f_{1}} \\
1+\hat{x}_{1}= & b_{1} \stackrel{(56)}{<} 1+x_{1} \xi \\
1+x_{2}= & \frac{1}{f_{2}} \stackrel{(57)}{<} 1+\hat{x}_{1} \xi \stackrel{(58)}{<} 1+x_{1} \xi^{2} \\
1+\hat{x}_{2}= & b_{2} \stackrel{(56)}{<} 1+x_{2} \xi \stackrel{(59)}{<} 1+x_{1} \xi^{3} \\
& \vdots \\
1+x_{n}= & \frac{1}{f_{n}} \stackrel{(57)}{<} 1+\hat{x}_{n-1} \xi<1+x_{1} \xi^{2 n-2} \\
1+\hat{x}_{n}= & b_{n} \stackrel{(56)}{<} 1+x_{n} \xi \stackrel{(60)}{<} 1+x_{1} \xi^{2 n-1} \quad \text { etc. }
\end{aligned}
$$

It follows that

$$
\begin{aligned}
p_{n+1}^{R} & =p_{1}^{R} \frac{1}{f_{1}} b_{1} \cdots \frac{1}{f_{n}} b_{n} \\
& =p_{1}^{R}\left(1+x_{1}\right)\left(1+\hat{x}_{1}\right) \cdots\left(1+x_{n}\right)\left(1+\hat{x}_{n}\right) \\
& <p_{1}^{R}\left(1+x_{1}\right)\left(1+x_{1} \xi\right) \cdots\left(1+x_{1} \xi^{2 n-2}\right)\left(1+x_{1} \xi^{2 n-1}\right) \\
& =p_{1}^{R} \prod_{m=0}^{2 n-1}\left(1+x_{1} \xi^{m}\right) .
\end{aligned}
$$

Since $\xi \in(0,1), \prod_{m=0}^{\infty}\left(1+x_{1} \xi^{m}\right)$ converges. This shows that the pressure in the solutions constructed above remains uniformly bounded in time. We summarize our findings in:

Proposition 4.3. Given the Euler system (1), (2), (3) describing one-dimensional, compressible flow of an ideal polytropic gas (7). Consider any Cauchy problem where the data produce two contact waves, together with an admissible shock between them, and where the density is lower in the region between the contacts than in the outer regions; see Figure 1.

Then, by imposing absorbing boundary conditions at fixed locations (in Lagrangian coordinates and depending on the data) to the left and to the right of the two contacts, the resulting flow is defined for all times and consists of only shocks and contacts. Furthermore, the pressure, density, particle velocities and shock speeds are all uniformly bounded in time, the bounds depending on the initial data and the given parameters $\gamma, K$.

\section{Scaling invariance of the 1-D Euler system}

In the analysis above we found it useful to parametrize wave curves by ratios of pressures and specific volumes. One reason to consider this choice for measuring wave strengths is the fact that the Euler system is formally invariant under the scaling

$$
(\tau, u, p, E) \mapsto\left(\zeta \tau, \zeta u, \zeta p, \zeta^{2} E\right) \quad \zeta>0,
$$


which preserves such ratios. While the four variables above are not independent, this scaling is consistent for ideal polytropic gases. More generally, the Euler system is invariant under the scaling $(\tau, u, p) \mapsto(\zeta \tau, \zeta u, \zeta p)$ provided the internal energy function $e(\tau, p)$ satisfies

$$
e(\zeta \tau, \zeta p)=\zeta^{2} e(\tau, p)
$$

This clearly holds for ideal polytropic gases (7). A direct calculation shows that if (61) holds, then the characteristic speeds $\lambda_{0}, \lambda_{ \pm}$, as well as shock speeds, are invariant as well.

It follows that if $U(x, t)=(\tau(x, t), u(x, t), p(x, t))$ is any (weak or classical) solution of the Euler system (1), (2), (3) with initial data $U_{0}(x)=\left(\tau_{0}(x), u_{0}(x), p_{0}(x)\right)$, then $V(x, t):=\zeta U(x, t)(\zeta>0)$ is again a solution with data $V_{0}(x)=\zeta U_{0}(x)$. Furthermore, the wave patterns and interactions are identical in the two solutions, and it is clear that $V(x, t)$ is an entropy admissible weak solution if and only if the same holds for $U(x, t)$. As a consequence we see that once we have a solution to the Euler system (with an equation of state satisfying (61)), then we can obtain other solutions with arbitrarily large (or small) amplitudes, and variations, by applying the scaling above.

As a final observation we note that it is natural to try and argue the other way as well. That is: given large BV data for the Euler system, we can scale these down ( $\zeta$ small) to obtain BV data with small variation; then apply Glimm's theorem to obtain a global-in-time weak solution; and finally scale up again (by $1 / \zeta$ ). However, this argument is flawed since the amount of variation in the data allowed by Glimm's theorem depend on where the data are located in $(\tau, u, p)$-space. In particular, as the set where the data lie shrinks towards the origin in $(\tau, u, p)$-space under the down-scaling, so does the amount of variation allowed by Glimm's theorem. Thus there is no guarantee that Glimm's theorem applies to the scaled-down data.

\section{References}

[1] N. S. Bahvalov, The existence in the large of a regular solution of a quasilinear hyperbolic system, Ž. Vyčisl. Mat. i Mat. Fiz. 10 (1970), 969-980 (Russian). MR 0279443 (43 \#5165)

[2] Stefano Bianchini, BV solutions of the semidiscrete upwind scheme, Arch. Ration. Mech. Anal. 167 (2003), no. 1, 1-81. MR 1967667 (2004k:35249)

[3] - Hyperbolic limit of the Jin-Xin relaxation model, Comm. Pure Appl. Math. 59 (2006), no. 5, 688-753. MR 2172805 (2008b:35167)

[4] Stefano Bianchini and Alberto Bressan, Vanishing viscosity solutions of nonlinear hyperbolic systems, Ann. of Math. (2) 161 (2005), no. 1, 223-342. MR 2150387 (2007i:35160)

[5] Alberto Bressan, Global solutions of systems of conservation laws by wave-front tracking, J. Math. Anal. Appl. 170 (1992), no. 2, 414-432. MR 1188562 (93k:35166)

[6] Tung Chang and Ling Hsiao, The Riemann problem and interaction of waves in gas dynamics, Pitman Monographs and Surveys in Pure and Applied Mathematics, vol. 41, Longman Scientific \& Technical, Harlow, 1989. MR 994414 (90m:35122)

[7] R. Courant and K. O. Friedrichs, Supersonic Flow and Shock Waves, Interscience Publishers, Inc., New York, N. Y., 1948. MR $0029615(10,637 \mathrm{c})$

[8] Ronald J. DiPerna, Existence in the large for quasilinear hyperbolic conservation laws, Arch. Rational Mech. Anal. 52 (1973), 244-257. MR 0338576 (49 \#3340)

[9] Global existence of solutions to nonlinear hyperbolic systems of conservation laws, J. Differential Equations 20 (1976), no. 1, 187-212. MR 0404872 (53 \#8672)

[10] Thaddeus J. Edens, Spectral analysis of a model of the compressible Euler equations, Commun. Math. Sci. 5 (2007), no. 1, 205-232. MR 2310640 (2008b:35172) 
[11] James Glimm, Solutions in the large for nonlinear hyperbolic systems of equations, Comm. Pure Appl. Math. 18 (1965), 697-715. MR 0194770 (33 \#2976)

[12] Helge Kristian Jenssen, Blowup for systems of conservation laws, SIAM J. Math. Anal. 31 (2000), no. 4, 894-908 (electronic). MR 1752421 (2001a:35114)

[13] J.-L. Joly, G. Métivier, and J. Rauch, A nonlinear instability for $3 \times 3$ systems of conservation laws, Comm. Math. Phys. 162 (1994), no. 1, 47-59. MR 1272766 (95f:35145)

[14] Tai Ping Liu, The deterministic version of the Glimm scheme, Comm. Math. Phys. 57 (1977), no. 2, $135-148$. MR 0470508 (57 \#10259)

[15] Solutions in the large for the equations of nonisentropic gas dynamics, Indiana Univ. Math. J. 26 (1977), no. 1, 147-177. MR 0435618 (55 \#8576)

[16] _ Initial-boundary value problems for gas dynamics, Arch. Rational Mech. Anal. 64 (1977), no. 2, $137-168$. MR 0433017 (55 \#5996)

[17] Takaaki Nishida, Global solution for an initial boundary value problem of a quasilinear hyperbolic system, Proc. Japan Acad. 44 (1968), 642-646. MR 0236526 (38 \#4821)

[18] Takaaki Nishida and Joel A. Smoller, Solutions in the large for some nonlinear hyperbolic conservation laws, Comm. Pure Appl. Math. 26 (1973), 183-200. MR 0330789 (48 \#9126)

[19] Takaaki Nishida and Joel Smoller, Mixed problems for nonlinear conservation laws, J. Differential Equations 23 (1977), no. 2, 244-269. MR 0427852 (55 \#882)

[20] Yue-Jun Peng, Solutions faibles globales pour l'équation d'Euler d'un fluide compressible avec de grandes données initiales, Comm. Partial Differential Equations 17 (1992), no. 1-2, 161-187 (French, with English summary). MR 1151259 (93e:35083)

[21] Nils Henrik Risebro, A front-tracking alternative to the random choice method, Proc. Amer. Math. Soc. 117 (1993), no. 4, 1125-1139. MR 1120511 (93e:35071)

[22] B. L. Roždestvenskiǔ and N. N. Janenko, Systems of quasilinear equations and their applications to gas dynamics, Translations of Mathematical Monographs, vol. 55, American Mathematical Society, Providence, RI, 1983. Translated from the second Russian edition by J. R. Schulenberger. MR 694243 (85f:35127)

[23] Yu. G. Rykov, On the Cauchy problem for systems of equations of one-dimensional gas dynamics, Tr. Mosk. Mat. Obs. 59 (1998), 221-239 (Russian, with Russian summary); English transl., Trans. Moscow Math. Soc. (1998), 201-216. MR 1824509 (2001k:76080)

[24] Denis Serre, Systems of conservation laws. 1, Cambridge University Press, Cambridge, 1999. Hyperbolicity, entropies, shock waves; Translated from the 1996 French original by I. N. Sneddon. MR 1707279 (2000g:35142)

[25] Joel Smoller, Shock waves and reaction-diffusion equations, 2nd ed., Grundlehren der Mathematischen Wissenschaften [Fundamental Principles of Mathematical Sciences], vol. 258, Springer-Verlag, New York, 1994. MR 1301779 (95g:35002)

[26] J. Blake Temple, Solutions in the large for the nonlinear hyperbolic conservation laws of gas dynamics, J. Differential Equations 41 (1981), no. 1, 96-161. MR 626623 (82i:35117)

[27] B. Temple and R. Young, The large time stability of sound waves, Comm. Math. Phys. 179 (1996), no. 2, $417-466$. MR 1400747 (97f:35132)

[28] Robin Young, Exact solutions to degenerate conservation laws, SIAM J. Math. Anal. 30 (1999), no. 3, 537-558 (electronic). MR 1677943 (2000b:35165) 